\title{
Cancer care in a time of COVID: lung cancer patient's experience of telehealth and connectedness
}

\author{
Anne Fraser ${ }^{1,2}\left(\mathbb{D} \cdot\right.$ Rob McNeill $^{2} \cdot$ Jackie Robinson $^{1,3}$ \\ Received: 2 May 2021 / Accepted: 29 August 2021 / Published online: 4 October 2021 \\ (c) The Author(s), under exclusive licence to Springer-Verlag GmbH Germany, part of Springer Nature 2021
}

\begin{abstract}
Objective To explore lung cancer patient's experiences of telehealth during COVID-19 restrictions.

Methods Thirty patients with lung cancer were recruited. Data was collected using a qualitative exploratory design with semi-structured interviews. Transcripts were thematically coded using NVivo software.

Results Five key themes were identified: maintaining resilience, participants acknowledged that they were self-reliant prior to their diagnosis and that the sense of their own internal capabilities was a source of comfort for them; importance of preestablished relationships with healthcare professionals, the sense of connection established prior to the telehealth consultation supported participants to engage with healthcare professionals where the need for connectedness was amplified by a sense of isolation; seeking help, participants sought help from services that they perceived as being "expert"; convenience, factors such as costs and saving time were highlighted; and preferences for consultation type, majority of participants identified physical and emotional comfort being in their own space. For a small number of patients, continuing a face-to-face assessment was important due to expectation based on previous experience.

Conclusion The use of telehealth was supported during the management of COVID-19. Connectedness and convenience were key to the level of comfort and confidence for patients with lung cancer using telehealth during "lockdown."
\end{abstract}

Keywords Lung cancer $\cdot$ COVID-19 $\cdot$ Surveillance $\cdot$ Follow-up $\cdot$ Resilience $\cdot$ Telehealth

\section{Introduction}

As healthcare systems have raced to prepare for, and manage, the SARS-CoV-2 (COVID19) pandemic, we have seen a reorganization of how healthcare is delivered. Delivery of cancer care has been affected globally [1-3]. New Zealand (NZ) entered alert level 3 on 23 March 2020, restricting nonessential travel, work, and school (https://covid19.govt.nz/ alert-system/about-the-alert-system/). On 25 March 2020, NZ moved to alert level 4 for five weeks, essentially locking down the country for all but essential services. People stayed home within their "bubbles" (immediate family groups),

Anne Fraser

ann@adhb.govt.nz

1 Auckland City Hospital, 2 Grafton Road, Auckland, New Zealand

2 School of Population Health, University of Auckland, Private Bag 92019, Auckland 1142, New Zealand

3 School of Nursing, University of Auckland, Private Bag 92019, Auckland 1142, New Zealand and healthcare delivery dramatically changed. People were instructed to stay at home in their "bubble" other than for essential personal movement; safe recreational activity was allowed close to home; travel was severely limited; all gatherings were cancelled, and all public venues closed; businesses were closed except for essential services; for example, supermarkets, pharmacies, clinics, petrol stations, and lifeline utilities stayed open; Educational facilities were closed; rationing of supplies and requisitioning of facilities was possible; Reprioritization of healthcare services began.

In NZ, we were afforded the benefit of learning from our colleagues around the world as they adapted rapidly to the barriers related to COVID-19 restrictions in delivering healthcare. Auckland Regional Cancer and Blood Service, which provides both oncology and hematology services for a population of approximately 700,000 , quickly implemented pathways to protect vulnerable patients. This included screening at the entrance to hospital buildings, restriction of visitors, delay of non-urgent investigations and treatment, application of PPE, and a shift from on-site clinic visits to telehealth. 
Where clinicians may have historically resisted alternatives to face-to-face clinic patient consultations, universally adopted moves to alternatives such as telehealth became common during the COVID-19 restrictions. While telehealth offers an alternative approach to care delivery, there are a number of factors for consideration. Literature describing the use of telehealth and video conferencing identifies advantages and disadvantages for patients and clinicians [4-13]. Virtual assessments may offer a reduction in patient related costs such as petrol, travel, time off work, and childcare. In addition, some patients report that a virtual assessment is less stressful than attending a face-to-face consultation [7]. Clinicians identified convenience and flexibility with virtual consultations and expressed that the addition of tools such as email and texting could facilitate assessments in the cases of patients who were too anxious to attend face-to-face consultations or had communication difficulties [5] However, concern regarding telehealth is expressed in a number of reviews, with the potential for increased clinical risk and future uptake of services. Challenges related to technology, including familiarity of use, access to internet and data, and confidentiality, are all ongoing concerns for services planning virtual assessments and remote care delivery [5]. Concerns on how to communicate and connect with patients are being addressed in the development of virtual conversation guides and resources [14-19].

The aim of this study was to explore lung cancer patient's experiences of telehealth consultations during COVID-19 restrictions.

\section{Methods}

\section{Design}

A qualitative exploratory design using semi-structured interviews to collect patient experiences of telehealth. An exploration of the barriers and enablers to telehealth assessments during COVID-19 lockdowns.

\section{Sampling and recruitment}

Thirty patients with lung cancer who had been registered with the oncology department during level 3 and level 4 lockdowns were recruited between May and June 2020. Participants were eligible if they had a diagnosis of lung cancer regardless of their current or expected illness trajectory. Patients were approached consecutively until ten slots were filled in each cohort. Due to restrictions around translation services, patients could not be recruited if they did not speak English fluently and had required telephone interpreters during telehealth assessments.
Purposeful sampling was used to ensure Māori patients were represented, in keeping with our commitments to the Treaty of Waitangi. The Treaty of Waitangi is New Zealand's founding document and forms an agreement between Māori and Pakeha (English) around the principles of partnership, participation, and protection [20].

Eligible participants' identified from electronic clinical records were invited to participate by an Oncology Research Nurse. People who expressed an interest to participate were emailed a participant information sheet and consent form. They were called a week later to obtain verbal consent.

\section{Data collection}

Semi-structured interviews were conducted over the telephone, using an interview guide to facilitate discussion (see Table 1). Interview questions were developed following a review of the telehealth literature and from information already gathered as part of a larger study on the use of remote surveillance models in lung cancer (yet to be published). Patient's whānau (extended family; sometimes used to include friends who may not have any kinship ties) were invited to contribute to the interviews. Interviews were recorded and transcribed verbatim (Table 2).

\section{Data analysis}

Interview transcripts were read and thematically coded using NVivo software, following a general inductive analysis approach [21]. An iterative process was applied across all codes, as themes were considered and developed into broader conceptual themes [21, 22]. Rigor was achieved through inter-rater reliability via independent review of codes by the co-authors, peer review and discussion of findings, and by selecting quotes which contained a rich description of the patient experience.

\section{Ethics}

Ethics was obtained from the Auckland Health Research Ethics Committee (AHREC AH1337).

\section{Findings}

Participants were predominantly female and of New Zealand European ethnicity, but distributed evenly across the treatment trajectory: curative intent such as adjuvant cisplatin and vinorelbine, palliative intent such as carboplatin and gemcitabine, and patients between treatments under surveillance. Māori indigenous peoples were purposefully recruited with the aim of having $25 \%$ representation. Participants were predominantly in the 60-69 years old age bracket (Table 2). 
Table 1 Interview guide

What did you feel were positive things about telehealth?

Did you feel your concerns and needs were covered?

How did being in your own environment impact your feelings of safety?

What concerns did you have not seeing someone face to face?

Would you prefer human contact?

What concerns, if any, did you have not having a physical exam?

How did you find the technology?

What was the burden at your end?

What happened for you between TH calls?

If you had symptoms that you were concerned about, what was your experience of contacting the service?

Do you feel you gained a sense of independence with your healthcare, and if so, how did that impact your confidence and independence around your health?

In what ways did you gain education about your healthcare, self-care, and management of your symptoms?
Did you feel less rushed than in the hospital setting?

Did telehealth feel less personal?

Technology, time, including whānau, utilizing interpreters? Cancer society, nurse specialist, acute oncology, ED, GP

Positive experiences and difficulties you had. Did you contact those support services more than usual?
Table 2 Sample summary

\begin{tabular}{ll}
\hline Gender & \\
Male & 11 \\
Female & 19 \\
Ethnicity & \\
NZ European & 18 \\
Māori & 6 \\
Cook Island Māori & 1 \\
Asian other & 1 \\
Pasifika (Samoan) & 2 \\
Asian & 1 \\
European Other & 1 \\
Intent to treat & \\
Curative & 10 \\
Palliative & 10 \\
Surveillance & 10 \\
Age & \\
$40-49$ & 1 \\
$50-59$ & 8 \\
$60-69$ & 13 \\
$70-79$ & 7 \\
$80+$ & 1 \\
\hline &
\end{tabular}

Five key themes:

- Convenience for patient and whānau

- Preferences for consultation types

- Importance of pre-established relationships with healthcare professionals

- Seeking help

- Maintaining resilience

\section{Convenience for patient and whānau}

Many participants talked about the convenience of consulting with healthcare professionals without leaving home. Factors such as saving time, travel costs, and not having to take time off work to attend hospital appointments were highlighted. In addition, many participants referred to barriers they had previously experienced when having to come to the hospital for appointments that no longer existed during telehealth consultations:

Truthfully, I felt it was easier instead of getting in a car and having to travel all the way to the hospital and sit and wait there for 30 minutes while I'm in a queue then go into an office for 10 minutes. The time saving alone was immense. (Female, NZE, Curative, 64)

For one participant the convenience of telehealth had an impact not only on herself but on the people she relied on to bring her to hospital since she could no longer drive:

With not being able to drive anymore, it was great that I didn't have to find somewhere that would take time, not just out of my day, but somebody else's day. And sometimes it can be a good half a day, just to attend maybe a 15-minute clinic. By the time you've got in and parked and prepared. So in terms of timing, it was really great, I really appreciated the fact that I could just pick up the phone and have a good conversation and put it down and get on with the rest of my day. (Female, NZE, Palliative, 72) 


\section{Preferences for consultation type}

Participants expressed a range of preferences around faceto-face assessments versus telehealth. For a small number of patients, continuing a traditional face-to-face assessment was important as they had an expectation based on previous experience. One participant identified that the healthcare system had always used face-to-face consultations as the standard approach:

It seems more personal with the doctor. You're so used to seeing your GP face-to-face that you've been used to for years and years. (Male, NZE, Palliative, 85)

Other participants described the importance of body language during face-to-face consultations and how this helped with communication:

I would prefer face-to-face, just because I think it's more personalised and I think you can gauge the person's body language that's talking to me, and they can see my body language and they can see my physical appearance. We're talking about illness, so sometimes what I say and what I see is totally different. (Female, NZE, Palliative, 67)

However, the majority of participants preferred telehealth, identifying both physical and emotional comfort being in their own space:

It's easier to speak when I'm not looking at the person, I can be a little bit more honest with myself and then have to go in and to have to worry about telling them this and that I didn't want to be there or I wasn't feeling well and I didn't want to see them. So, it was easier just to do the interview on the phone. (Female, Maori, Surveillance, 59)

A sense of physical safety not having to go to hospital for consultations was raised by a number of participants, and this impacted on their preference:

Having sat in the waiting room several times in clinics and stuff, you can be there for quite a long time and there's people around you with lots of different things going on so a lot of germs and whatnot. I think it's healthier to just take a call at home. (Female, NZE, Curative, 64)

\section{Importance of established relationships prior to COVID-19}

A strong preference for well-established relationships permeated the findings. The sense of connection established prior to the telehealth consultation supported participants to engage with healthcare professionals. Many participants identified the importance of connectedness during COVID19 , which was amplified by a sense of isolation and lack of perceived access to support services:

Ifound it easier actually talking to the people that I've built a relationship with, than talking to a voice over a phone.... you never know what you're going to get told. And so, meeting someone new on the phone didn't work as well for me as going in, seeing the people face-to-face that I know, they make me feel relaxed and I can talk to them quite easily. (Female, Māori, Palliative, 62)

Many participants recognized that it was easier to feel a sense of security in the new process if they could connect with team members they could identify over the telephone:

I suppose because I've been under these doctors for quite a while now and you sort of get a trust with them. (Female, NZE, Curative, 60)

\section{Seeking help during telehealth and COVID restrictions}

Participants described various ways they sought help during the COVID-19 restrictions, including accessing hospital and community based support, and online information.

Motivation to contact certain services varied. For example, for some, participants sought help from services that they perceived as being the "experts":

The doctors knew what I was talking about and they gave me something or told me what to do. Like, if my temperature got too high, or I got shivers again, to ring up to them and then go to the hospital. (Female, NZE, Surveillance, 74)

In part, the sense of expertise came from "conditioning" during orientation to oncology services, and therefore strong relationships with hospital based support did not alter during COVID-19 restrictions:

I know everybody well enough that if I am in trouble with something, I can reach out and ask about that straight away. (Male, Samoan, Palliative, 49)

Participants who were well acquainted with the oncology system sought hospital-based resources, such as their clinical nurse specialist (CNS). Participants described a sense of familiarity and confidence in this relationship and this method of care delivery, which played an important role for patients developing resilience during COVID-19 management:

T' [CNS] at Oncology in Auckland, if I'd ring and leave her a message, just because I always automati- 
cally ring people first, or I'd send her a text and she'd ring me back as soon as she could. (Male, NZ European, Curative, 68)

Contact through the oncology department allowed participants to be triaged over the telephone and have medication scripts emailed out to community pharmacies. Remote management was more convenient than attending their family physician, as no appointments were required, and they were accessing oncology expertise directly:

The doctors knew what I was talking about and they gave me something or told me what to do. Like, if my temperature got too high, or I got shivers again, to ring up to them and then go to the hospital. It was actually good talking to them over the phone. (Female, NZ European, Surveillance, 58)

Participants reported having regular phone calls from their community oncology nurse, who were able to help them with clinical concerns, prescriptions, and palliative care support. Again, well-established relationships with staff which participants perceived as experts played a reassuring role during the period of telehealth assessments:

I had one of the Cancer Society community nurses who was ringing me every week, just to make sure how I was. So, she was wonderful. (Female, Cook Island Māori, Curative, 55)

Some participants utilized online resources from websites that had been identified as useful by healthcare professionals. Participant described seeking information about managing side effects of treatment or on how to maximize their quality of life:

There were some pointers to some good websites, Macmillan et cetera, that were also really helpful. I'm also the type of person who likes to Google, sometimes overly so, but there's lots of information out there on the internet, you do have to be careful what you select. (Male, NZ European, Curative, 59).

\section{Resilience during telehealth and COVID-19 restrictions}

Many participants expressed a growing sense of independence during the COVID-19 restrictions and found various ways to build resilience, while they "got used to" a new normal:

$D$ rung them but the questions were answered straight away. So, there was no problem. I would maybe not have done that, if I had had to come into the hospital, I might've waited until I came in to ask the doctor. (Female, NZ European, Curative, 69)
Many participants acknowledged that they were already self-reliant prior to their diagnosis and that the sense of their own internal capabilities was a source of comfort for them:

I do try not to rely on people, which some people find quite annoying. (Female, NZ European, curative, 75)

I just bit the bullet and went along with what was happening. (Male, NZ European, Surveillance, 61)

Other participants expressed fear, isolation, and doubt during this period of time, which was influenced by relationships with whānau and friends:

I was really uptight because I was on my own, I had no support, I couldn't have my family with me. It was just super scary to be honest. (Female, NZ European, Palliative, 67)

A small number of participants were concerned to access support as they believed that the health service was already "overwhelmed" managing COVID issues".

I found it quite positive and I was quite pleased that I didn't have to go into the hospital in among all those people. (Female, Indian, Palliative, 69)

Participants identified a period of adjustment transitioning to telehealth assessments. A sense of confidence increased once participants had experienced their first call:

When I was told it's a phone assessment, talking to the doctor, instead of going there, I felt a little bit of unease, because it's a new sort of thing. But after the first phone assessment, from then I was all good. (Female, Māori, Palliative, 67)

But they were all really good at what they did and they explained the situation really well and sort of put you at ease. So, you didn't feel like you weren't getting the full picture. (Male, Samoan, Palliative, 49)

Participants identified confidence in the telehealth process; however, this was impacted by concerns around communication. Confidence is a key factor in building resilience, and the importance of communication during COVID-19 restrictions was raised by participants. There was fear that clinicians would not understand the symptoms being described; that they themselves may not understand communications from the oncology team; and fear around how they may have coped if they were told their cancer was progressing:

But that was my main concern, whether they could understand what I was trying to say properly. (Female, NZ European, Surveillance, 58)

A couple of the doctors, their foreign English was a little bit difficult to pick up over the telephone. (Male, NZ European, Surveillance, 61) 
I don't know how I'd find it if they found something else wrong, I don't know how I'd react to that. (Female, Māori, Surveillance, 58)

More positively, many participants described how telehealth gave them a chance to include whānau in a way they were unable to with on-site clinic visits. Participants described difficulties in coordinating whānau to attend a clinic appointment. However, with telehealth and family being at home during level 3 and 4 COVID-19 restrictions, involving them in the telehealth consultations was easier:

\section{I think that in the interviews there were opportunities to ask a lot of questions, I had support and in many of those interviews I had wither my wife with me, or my daughter with me, and they'd written things down that I had wanted to ask beforehand. (Male, NZE, Cura- tive, 59)}

\section{Discussion}

This study is the first conducted in NZ exploring experiences of lung cancer patients during level 4 COVID-19 restrictions. While there are a growing number of publications exploring or describing various aspects of telehealth during the COVID-19 pandemic globally, currently, none describes the patient experience and any barriers or enablers to carrying out telehealth assessments during this time. Participants in this study expressed a range of views associated with using telehealth. Most found the use of telehealth acceptable. For some participants, telehealth was preferable to usual clinic attendance. Participants expressed anxiety about visiting the hospital during COVID-19 restrictions, for fear of contracting COVID-19, or being around other sick patients.

While a number of themes relating to patient experience of telehealth are well described in the literature $[6,8,12$, 23, 24], two new themes emerged in this study: how patients seek help and resilience.

A systematic review found that patients using telehealth were reassured by a sense of having a safety net, with timely access to healthcare providers (HCP) [25]. Such a safety net may be considered active (where patients made active connections with HCP) or passive (where patients were being monitored passively by their HCP). Findings from this study suggests that how patients chose to seek help during COVID-19 telehealth did not vary hugely from their usual routine, with patients tending towards making contact where they had already established relationships, or where had built a sense of trust around perceptions of expertise. However, some participants found that their sense of independence grew and that they managed their own healthcare in ways they may not have prior to COVID-19 management, becoming less passive. Established relationships, ease of access to support, and communication were key values for patients when seeking help.

A sense of personalized care can play an important role in how patients engage with telehealth and in managing their own health care [23, 26]. In this study, having a range of options for patients to choose how they accessed support gave them a sense of personalized care, even in this pandemic setting. Less well understood is the concept of orientating patients in healthcare settings [25]. Patients are orientated at the start of oncology treatment and orientated to who, how, and when they should seek help. Due to the serious nature of side effects from oncology treatments, clinicians reinforce the importance of contacting the oncology department, rather than a family physician. Findings from this study suggest that during the COVID-19 restrictions, this heightened sense of needing to seek "expert help" from oncology services was exacerbated. In addition, the messages being relayed through the media around "vulnerable populations" including those having cancer treatment may have resulted in greater contact with "oncology experts."

The fear of contracting COVID-19 from a community source was a factor in decisions regarding contact with the family physician; however, factors such as inconvenience and associated costs may also be relevant. These issues may influence a patient's need to seek help from a trusted source. Similar to findings in this study, the importance of relationships with healthcare providers is identified in the literature $[6,27]$. Maintaining key relationships to adapt to a "new normal" may be utilized alongside personal resources and psychological tools to develop and maintain resilience. Participants in this current study identified the challenges of developing a trusted relationship with a member of the oncology team if they had not previously met them.

Resilience associated with the use of telehealth has not been well explored in the literature. However, a recent literature review identified a number of factors which contribute to a sense of resilience for cancer patients [28]. While a diagnosis of cancer may feel overwhelming, it can give patients the opportunity to develop personal skills and better coping strategies. Personal attributes such as "hardiness," which may be developed as a coping strategy, are important buffers for cancer patients [29, 30]. Family and friends are often a source of support and are important in making meaning of the cancer process [27, 28]. However, barriers exist which exclude family from outpatient clinic consultations including inability to get time away from work to attend appointments, the size of clinic rooms precluding more than one support person to attend, or the resistance to patients calling family members to "listen in" on the consultation. Participants in the current study identified similar concerns, stating that during telehealth, they could coordinate whannau involvement. Furthermore, being at home in a familiar 
surrounding helped participants to feel relaxed, whereas the clinic environment could be intimidating or unsupportive of cultural needs.

Strengths of this study are that the sample represented a range of gender, ethnicity, stages of disease, and ages. In addition, the interview questions were informed by previous literature on telehealth. Patient experiences informed findings, and the interviews took place during COVID-19 restrictions.

Numbers within each treatment intent group were small, and therefore it may not be possible to draw firm conclusions from the data gathered. The period of time patients had experienced telehealth assessments was also relatively short, and patient's experiences may alter over time, as they get used to telehealth appointments. Whilst New Zealand has been identified as a country that was more successful at managing waves of the virus, in part that was due to far more rigorous lockdown plans than other countries. Despite these restrictions being strictly enforced, telehealth ensured that the majority of cancer care could continue and could be used as a model for countries with healthcare systems overrun with COVID-19 support. A further limitation was the exclusion of patients who could not speak English and required translators. The group this impacted were Cantonese and Mandarin speaking patients, and if there was to be further expansion of this work, this would need addressing.

Future research including quality of life data would be useful to compare patient's understanding of resilience, how they maintained resilience, and the impact such active coping had on their quality of life. Follow-up of the impact of telehealth on cancer care beyond COVID-19 management would be useful to explore once there is a return to a new "normal." Currently in our own local practice, there has been a push from clinicians and systems to return to faceto-face assessments, which is being net with mixed response from patients. Many patients in our lung cancer clinics are requesting that they continue with telehealth assessments now where systems and processes are in place.

\section{Conclusions}

This study supports the use of telehealth during the management of the COVID-19 pandemic in the setting of lung cancer. The strength of relationships developed between healthcare providers, both within the hospital setting and the community, were key to this level of comfort and confidence. Participants identified the convenience of telehealth and a level of safety. Further research including a range of different cancer types may help to better understand relationships between active coping, resilience, and quality of life in the setting of telehealth.
Author contribution Study concept and design, AF/RM. Data collection and analysis, AF. Draft, AF. Revision of manuscript, AF/RM/JR.

Funding The research leading to these results received funding from the Cancer Society of New Zealand under Grant Agreement as a Research Training Grant.

Availability of data and material The data that support the findings of this study are available on request from the corresponding author. The data are not publically available due to privacy or ethical restrictions.

Code availability Not applicable.

\section{Declarations}

Ethics approval AH1337 Auckland Health Research Ethics Committee (AHREC).

Consent to participate Informed consent was obtained from all individual participants included in the study.

Consent for publication The participants have consented to the submission of the case report to the journal.

Conflict of interest The authors declare no competing interests.

\section{References}

1. Mayor S (2020) COVID-19: impact on cancer workforce and delivery of care. Lancet Oncol 21:633

2. Richards M, Anderson M, Carter P, Ebert BL, Mossialos E (2020) The impact of the COVID-19 pandemic on cancer care. Nature Cancer 1:565-567

3. Schrag D, Hershman DL, Basch E (2020) Oncology practice during the COVID-19 pandemic. JAMA 323:2005-2006

4. Atherton H, Brant H, Ziebland S, Bikker A, Campbell J, Gibson A, McKinstry B, Porqueddu T, Salisbury C (2018) Alternatives to the face-to-face consultation in general practice: focused ethnographic case study. Br J Gen Pract 68:e293-e300

5. Chi N-CD, George (2015) A systematic review of telehealth tools and interventions to support family caregivers. J Telemed telecare 21:37-44

6. Cox ALG, Marcu A, Piano M, Grosvenor W, Mold F, Maguire R, Ream E (2017) Cancer survivors' experience with telehealth: a systematic review and thematic synthesis. J Med Internet Res 19:e11

7. Greenhalgh T, Koh GCH, Car J (2020) Covid-19: a remote assessment in primary care. BMJ (Clinical research ed) 368: m1182

8. McLean S, Protti D, Sheikh A (2011) Telehealthcare for long term conditions. BMJ (Clinical research ed) 342: d120

9. McLean S, Sheikh A, Cresswell K, Nurmatov U, Mukherjee M, Hemmi A, Pagliari C (2013) The impact of telehealthcare on the quality and safety of care: a systematic overview. PLOS ONE 8:e71238

10. Sood S, Mbarika V, Jugoo S, Dookhy R, Doarn CR, Prakash N, Merrell RC (2007) What is telemedicine? A collection of 104 peer-reviewed perspectives and theoretical underpinnings. Telemedicine journal and e-health : the official journal of the American Telemedicine Association 13:573-590

11. Kahn JM (2015) Virtual visits - confronting the challenges of telemedicine. N Engl J Med 372:1684-1685 
12. Head BA, Keeney C, Studts JL, Khayat M, Bumpous J, Pfeifer M (2011) Feasibility and acceptance of a telehealth intervention to promote symptom management during treatment for head and neck cancer. The Journal of Supportive Oncology 9:e1

13. Greenhalgh T, Vijayaraghavan S, Wherton J, Shaw S, Byrne E, Campbell-Richards D, Bhattacharya S, Hanson P, Ramoutar S, Gutteridge C, Hodkinson I, Collard A, Morris J (2016) Virtual online consultations: advantages and limitations (VOCAL) study. BMJ Open 6:e009388

14. Gippsland Region Palliative Care Consortium (2015) http:// www.grpcc.com.au/healthprofessionals/resources/communicat ion-skills/

15. Ministry of Health (2020) https://www.health.govt.nz/our-work/ diseases-and-conditions/covid-19-novel-coronavirus/covid-19information-health-professionals/telehealth-and-online-tools

16. Greenhalgh T, Koh GCH, Car J (2020) Covid-19: A remote assessment in primary care. BMJ (Clin Res Ed) 368:m1182

17. Hospice New Zealand (2020) Communication challenges and COVID - 19. https://www.hospice.org/nz/wpcontent/uploads/ 2019/04/1-Phone-consultations-COVID-19-resource-V2-23August-2020-1-1.pdf

18. National Institute for Health and Care Excellence (2020) Specialty guides for patient management during the coronavirus pandemic: Clinical guide for the management of remote consultations and remote working in secondary care during the coronavirus pandemic. https://www.nice.org/uk/media/default/about/covid-19/ specialty-guides/specialty-guide-virtual-working-and-coronavirus.pdf

19. Vitaltalk (2020) COVID-19 Communication skills. https://www. vitaltalk.org/guides/covid-19-communication-skills/

20. History NZ (2017) The Treaty in brief'. https://nzhistory.govt.nz/ politics/treaty/the-treaty-in-brief, (Ministry for Culture and Heritage), updated 17-May-2017

21. Braun V, Clarke V (2006) Using thematic analysis in psychology. Qual Res Psychol 3:77-101
22. Morse JM (2015) Critical analysis of strategies for determining rigor in qualitative inquiry. Qual Health Res 25:1212-1222

23. Anna Cox EB, Cockle-Hearne J, Knibb W, Potter C, Faithfull S (2008) Nurse led telephone follow up in ovarian cancer: a psychosocial perspective. Eur J Oncol Nurs 12:412-417

24. Stacey D, Green E, Ballantyne B, Skrutkowski M, Whynot A, Tardif L, Tarasuk J, Carley M (2016) Patient and family experiences with accessing telephone cancer treatment symptom support: a descriptive study. Support Care Cancer 24:893-901

25. Chan RJ WJ, Marquart L. ( 2011) Information interventions for orienting patients and their carers to cancer care facilities. Cochrane Database of Systematic Reviews

26. Beaver K, Williamson S, Chalmers K (2010) Telephone follow-up after treatment for breast cancer: views and experiences of patients and specialist breast care nurses. J Clin Nurs 19:2916-2924

27. Molina Y, Yi JC, Martinez-Gutierrez J, Reding KW, Yi-Frazier JP, Rosenberg AR (2014) Resilience among patients across the cancer continuum: diverse perspectives. Clin J Oncol Nurs 18:93-101

28. Seiler A, Jenewein J (2019) Resilience in Cancer Patients Frontiers in Psychiatry 10

29. Aflakseir A, Nowroozi S, Mollazadeh J, Goodarzi MA (2016) The role of psychological hardiness and marital satisfaction in predicting posttraumatic growth in a sample of women with breast cancer in Isfahan. Iranian Journal of Cancer Prevention 9:e4080

30. Hamama-Raz Y (2012) Does psychological adjustment of melanoma survivors differs between genders? Psychooncology $21: 255-263$

Publisher's note Springer Nature remains neutral with regard to jurisdictional claims in published maps and institutional affiliations. 